\title{
Common Fixed Point Theorems for Hybrid Pairs of Mappings using Implicit Relations
}

\author{
Bhavana Deshpande and Rohit Pathak
}

\begin{abstract}
In this paper, we prove coincidence and fixed point theorems for two hybrid pairs of single valued and multivalued mappings on noncomplete metric spaces. We prove our results without using conditions of compatibility and continuity of mappings. We also give an example to validate our result.
\end{abstract}

\section{INTRODUCTION}

Jungck [5] generalized the concept of weak commutativity introduced by Sessa [24]. He defined the notion of compatible maps in order to generalize the concept of weak commutativity and showed that the weakly commuting maps are compatible but the converse is not true. The concept of weakly commuting mappings was extended by Kaneko [6] for multivalued set up and extended the result of Jungck [4]. Kaneko and Sessa [7] extended the concept of compatible mappings for multivalued mappings and generalized the result of Kubiak [26].

Nadlar [17] published a paper on multivalued mappings. Since then, the fixed point theory for single valued and multivalued mappings has been studied extensively and applied to diverse problems. This theory provides techniques for solving a variety of applied problems in mathematical science and engineering $[19,23]$. A number of generalization of Nadlar's results have appeared.

The study of noncompatible mappings was initiated by Pant [13-16]. He introduced $R$-weakly commutativity of mappings and compared $R$-weak commutativity and weak compatibility for single valued mappings. He showed that both $R$-weak commutativity and weak compatibility for single valued mappings are equivalent at the coincidence points. This idea of $R$-weak commutativity was independently extended to the settings of single valued and multivalued mappings by Shahzad and Kamran [12], Singh and Mishra [22].

2010 Mathematics Subject Classification. 54H25, 47H10.

Key words and phrases. Coincidence point, implicit relation, fixed point, compatible maps, property (E.A), (IT)-commuting maps, T-weakly commuting maps. 
Recently, Aamri and Moutawakil [10] defined a property (E.A) for self maps which contains the class of noncompatible maps. They obtained some fixed point theorems for such mappings using property (E.A) under strict contractive conditions.

Kamran [25] extended property (E.A) in the settings of single valued and multivalued mappings and generalized the notion of $(I T)$-commutativity for such pairs. He introduced the notion of $T$-weakly commuting map and showed that for hybrid pairs of mappings, (IT)-commuting at coincidence points implies $T$-weakly commuting but the converse is not true. He also showed that for single valued mappings $T$-weak commutativity at the coincidence points is equivalent to the weak compatibility.

Many authors have proved fixed and coincidence point theorems for hybrid pairs of mappings including Asad and Ahmad [1], Fisher [2], Kubiaczyk and Deshpande [8], Sharma and Deshpande [20], Kaneko and Sessa [7], Kamran [25], Shahzad and Kamran [12], Sharma et al. [21].

We have observed that proving fixed point theorems using an implicit relation is a good idea since it covers several contractive conditions rather than one contractive condition (see [11]).

In this paper, we prove coincidence point and fixed point theorems for hybrid pairs of single valued and multivalued mappings on noncomplete metric spaces. The mappings involved in our results are noncompatible and discontinuous. We also give an example to validate our main result. We improve and extend the result of Kamran [25].

\section{Preliminaries}

Let $(X, d)$ be a metric space and suppose that $C B(X)$ denotes the set of non-empty, closed and bounded subsets of $X$.

For $A, B \in C B(X)$, we denote

$$
\begin{aligned}
D(A, B) & =\inf \{d(a, b): a \in A, b \in B\} \\
D(x, A) & =\inf \{d(x, a): a \in A\} \\
H(A, B) & =\max \{\sup \{D(a, B): a \in A\}, \sup \{D(A, b): b \in B\}\}
\end{aligned}
$$

It is well known that $(C B(X), H)$ is a metric space with the distance function $\mathrm{H}$. Moreover, $(C B(X), H)$ is complete in the event that $(X, d)$ is complete.

Definition 1 ( [6]). Let $(X, d)$ be a metric space, $F: X \rightarrow C B(X)$ and $T: X \rightarrow X$. Then the pair $\{F, T\}$ is said to be weakly commuting if for each $x \in X, T F(x) \in C B(X)$ and $H(F T x, T F x) \leq D(T x, F x)$.

Definition 2 ( [7]). Let $(X, d)$ be a metric space, $F: X \rightarrow C B(X)$ and $T: X \rightarrow X$. Then the pair $\{F, T\}$ is said to be compatible if and only if $T F x \in C B(X)$ for each $x \in X$ and $H\left(F T x_{n}, T F x_{n}\right) \rightarrow 0$, whenever $\left\{x_{n}\right\}$ is a sequence in $X$ such that $F x_{n} \rightarrow M \in C B(X)$ and $T x_{n} \rightarrow t \in M$. 
Definition 3 ( [10]). The maps $f: X \rightarrow X$ and $g: X \rightarrow X$ are said to satisfy the property $(E . A)$ if there exists a sequence $\left\{x_{n}\right\}$ in $X$ such that $\lim _{n \rightarrow \infty} f x_{n}=\lim _{n \rightarrow \infty} g x_{n}=t \in X$.

Definition 4 ( [25]). The maps $f: X \rightarrow X$ and $T: X \rightarrow C B(X)$ are said to satisfy the property $(E . A)$ if there exists a sequence $\left\{x_{n}\right\}$ in $X$ such that $\lim _{n \rightarrow \infty} f x_{n}=t \in A=\lim _{n \rightarrow \infty} T x_{n}$ for some $t \in X$ and $A \in C B(X)$.

Definition 5 ( [18]). The mappings $T: X \rightarrow X$ and $F: X \rightarrow C B(X)$ are said to be $(I T)$-commuting at $x \in X$ if $T F x \subseteq F T x$.

Definition 6 ( [25]). Let $T: X \rightarrow C B(X)$, The map $f: X \rightarrow X$ is said to be $T$-weakly commuting at $x \in X$ if $f f x \in T f x$.

Example 1. Let $X=(0, \infty)$ with the usual metric $d$. Define $f: X \rightarrow X$ and $F: X \rightarrow C B(X)$ by $f x=4 x$ and $F x=[0,2+4 x]$ for all $x \in X$. Consider the sequence $\left\{x_{n}\right\}$ in $X$ given by $x_{n}=1+\frac{1}{n}, n=1,2,3, \ldots$

Then $\lim _{n \rightarrow \infty} f x_{n}=4 \in[0,6]=\lim _{n \rightarrow \infty} F x_{n}$ and $\lim _{n \rightarrow \infty} H\left(f F x_{n}, F f x_{n}\right) \neq 0$.

Therefore, $f$ and $F$ satisfy property (E.A), but they are not compatible.

Also for all $x \in X, f x \in F x, f f x=16 x \in F f x=[0,2+16 x]$. Therefore $f$ is $F$-weakly commuting.

Further, $f F x=[0,8+16 x] \nsubseteq F f x=[0,2+16 x]$. Therefore $f$ and $F$ are not $(I T)$-commuting. Also note that $f$ and $F$ are not weakly compatible.

Example 2. Let $X=[0,1)$ with the usual metric $d$. Define $f: X \rightarrow X$ and $F: X \rightarrow C B(X)$ by $f x=\frac{x}{2}$ and $F x=[0, x]$ for all $x \in X$. Consider the sequence $\left\{x_{n}\right\}$ in $X$ given by $x_{n}=\frac{n-1}{2(n+1)}, n=1,2,3, \ldots$

Then $\lim _{n \rightarrow \infty} f x_{n}=\frac{1}{4} \in\left[0, \frac{1}{2}\right]=\lim _{n \rightarrow \infty} F x_{n}$ and $\lim _{n \rightarrow \infty} H\left(f F x_{n}, F f x_{n}\right)=0$.

Therefore, $f$ and $F$ satisfy property $(E . A)$ and the hybrid pair $\{f, F\}$ is compatible. Also for all $x \in X, f x \in F x, f f x=\frac{x}{4} \in F f x=\left[0, \frac{x}{2}\right]$. Therefore $f$ is $F$-weakly commuting. Also the pair $\{f, F\}$ is $(I T)$-commuting because $f F x \subseteq F f x$.

\section{Implicit Relations}

Let $\Phi$ be the family of all continuous maps $\varphi: \mathbb{R}_{+}^{6} \rightarrow \mathbb{R}$ such that:

$\left(\varphi_{1}\right) \varphi$ is non-increasing in variable $t_{6}$.

$\left(\varphi_{2}\right)$ there exists $h \in(0,1)$ such that for all $u, v \geq 0$ with

$\left(\varphi_{a}\right): \varphi(u, v, v, u, u+v, 0) \leq 0$, or

$\left(\varphi_{b}\right): \varphi(u, v, u, v, 0, u+v) \leq 0$,

then we have $u \leq h v$.

$\left(\varphi_{3}\right) \varphi(u, u, 0,0,0, u)>0$ for all $u>0$. 


\section{Example 3.}

$\varphi\left(t_{1}, t_{2}, t_{3}, t_{4}, t_{5}, t_{6}\right)=t_{1}-\alpha \max \left\{\frac{t_{2}^{2}+t_{3}^{2}}{t_{2}+t_{3}},\left(t_{5}+t_{6}\right), t_{4}\right\}, \quad \alpha \in\left(0, \frac{1}{2}\right)$.

$\left(\varphi_{1}\right)$ Obviously.

$\left(\varphi_{2}\right)$ Let $u, v \geq 0$, then

$$
\begin{aligned}
\left(\varphi_{a}\right) \varphi(u, v, v, u, u+v, 0) & =u-\alpha \max \{v,(u+v), u\} \\
& =u-\alpha(u+v) \\
& =(1-\alpha) u-\alpha v
\end{aligned}
$$

Therefore

$$
\varphi(u, v, v, u, u+v, 0) \leq 0 \Rightarrow u \leq \frac{\alpha}{(1-\alpha)} v=h v,
$$

where $h=\frac{\alpha}{(1-\alpha)}, 0<h<1$.

$\left(\varphi_{3}\right) \varphi(u, u, 0,0,0, u)=u-\alpha \max \{u, u\}=(1-\alpha) u>0$ for all $u>0$.

\section{Example 4.}

$$
\varphi\left(t_{1}, t_{2}, t_{3}, t_{4}, t_{5}, t_{6}\right)=t_{1}-\alpha \frac{\max \left\{t_{4}-t_{2}, t_{4}-t_{3}\right\}}{1+t_{5} t_{6}}-(1-\alpha) t_{5} t_{6}
$$

where $\alpha \in(0,1), a, b>0, a+b<1$.

$\left(\varphi_{1}\right)$ Obviously.

$\left(\varphi_{2}\right)$ Let $u, v \geq 0$, then

$$
\begin{aligned}
\left(\varphi_{a}\right) \varphi(u, v, v, u, u+v, 0) & =u+\alpha(u-v) \\
& =(1+\alpha) u-\alpha v \leq 0 \\
& \Rightarrow u \leq \frac{\alpha}{1+\alpha} v=h v,
\end{aligned}
$$

where $h=\frac{\alpha}{1+\alpha}, 0<h<1$.

$$
\left(\varphi_{3}\right) \varphi(u, u, 0,0,0, u)=u+\alpha u>0 \text { for all } u>0 .
$$

\section{Example 5.}

$$
\varphi\left(t_{1}, t_{2}, t_{3}, t_{4}, t_{5}, t_{6}\right)=t_{1}^{p}-\left(\alpha t_{2}^{p}+\beta\left(t_{3}^{p}-t_{4}^{p}\right)\right)\left(1+t_{5} t_{6}\right)
$$

where $\alpha, \beta \in(0,1), \alpha+\beta<1$ and $p$ is an integer such that $p \geq 1$.

$\left(\varphi_{1}\right)$ Obviously.

$\left(\varphi_{2}\right)$ Let $u, v \geq 0$, then

$$
\begin{aligned}
\left(\varphi_{a}\right) \varphi(u, v, v, u, u+v, 0) & =u^{p}-\left(\alpha v^{p}+\beta\left(v^{p}-u^{p}\right)\right) \\
& =(1+\beta) u^{p}-(\alpha+\beta) v^{p} \\
& \leq 0 \\
& \Rightarrow u \leq\left(\frac{\alpha+\beta}{1+\beta}\right)^{1 / p} v=h v
\end{aligned}
$$

where $h=\left(\frac{\alpha+\beta}{1+\beta}\right)^{1 / p}, 0<h<1$.

$\left(\varphi_{3}\right) \varphi(u, u, 0,0,0, u)=u^{p}-\alpha u^{p}=(1-\alpha) u^{p}>0$ for all $u>0$. 


\section{Example 6.}

$$
\varphi\left(t_{1}, t_{2}, t_{3}, t_{4}, t_{5}, t_{6}\right)=2 t_{1}-\frac{\max \left\{t_{2}, t_{4}, t_{5}\right\}}{1+\left(t_{2}-t_{3}\right) t_{4}}+a t_{5}-t_{3} t_{6},
$$

where $a \in(0,1)$.

$\left(\varphi_{1}\right)$ Obviously.

$\left(\varphi_{2}\right)$ Let $u, v \geq 0$, then

$$
\begin{aligned}
\left(\varphi_{a}\right) \varphi(u, v, v, u, u+v, 0) & =2 u-(u+v)+a(u+v) \\
& =(1+a) u-(1-a) v \\
& \leq 0 \\
& \Rightarrow u \leq \frac{1-a}{1+a} v=h v,
\end{aligned}
$$

where $h=\frac{1-a}{1+a}, 0<h<1$.

$$
\left(\varphi_{3}\right) \varphi(u, u, 0,0,0, u)=2 u-u=u>0 \text { for all } u>0 .
$$

\section{Example 7 .}

$$
\varphi\left(t_{1}, t_{2}, t_{3}, t_{4}, t_{5}, t_{6}\right)=\beta t_{1}^{p}+\frac{\alpha \max \left\{\left(t_{2}^{p}-t_{3}^{p}\right), t_{4}^{p}\right\}}{1+\left(\alpha t_{4}^{p}+\beta t_{5}^{p}\right)\left(t_{2}^{p}-t_{3}^{p}\right)}-\beta\left(t_{3}^{p}+t_{6}^{p}\right),
$$

where $\beta \in(0,1), \alpha \geq 1$ and $p$ is an integer such that $p \geq 1$.

$\left(\varphi_{1}\right)$ Obviously.

$\left(\varphi_{2}\right)$ Let $u, v \geq 0$, then

$$
\begin{aligned}
\left(\varphi_{a}\right) \varphi(u, v, v, u, u+v, 0) & =\beta u^{p}+\alpha u^{p}-\beta v^{p} \\
& =(\beta+\alpha) u^{p}-\beta v^{p} \\
& \leq 0 \\
& \Rightarrow u \leq\left(\frac{\beta}{\beta+\alpha}\right)^{1 / p} v=h v,
\end{aligned}
$$

where $h=\left(\frac{\beta}{\beta+\alpha}\right)^{1 / p}, 0<h<1$.

$$
\left(\varphi_{3}\right) \varphi(u, u, 0,0,0, u)=\beta u^{p}+\alpha u^{p}-\beta u^{p}=\alpha u^{p}>0 \text { for all } u>0 .
$$

\section{Example 8.}

$$
\varphi\left(t_{1}, t_{2}, t_{3}, t_{4}, t_{5}, t_{6}\right)=t_{1}-q \max \left\{t_{2}, \frac{t_{3}+t_{4}}{2}, \frac{t_{5}+t_{6}}{2}\right\},
$$

where $q \in(0,1)$.

$\left(\varphi_{1}\right)$ Obviously.

$\left(\varphi_{2}\right)$ Let $u, v \geq 0$, then

$$
\left(\varphi_{a}\right) \varphi(u, v, v, u, u+v, 0)=u-q \max \left\{v, \frac{v+u}{2}, \frac{u+v}{2}\right\} .
$$

If $\max \left\{v, \frac{v+u}{2}, \frac{u+v}{2}\right\}=v$, then

$$
\varphi(u, v, v, u, u+v, 0) \leq 0 \Rightarrow u \leq q v=h v,
$$

where $h=q, 0<h<1$. 


$$
\begin{aligned}
& \text { If } \max \left\{v, \frac{v+u}{2}, \frac{u+v}{2}\right\}=\frac{v+u}{2}, \\
& \qquad \begin{aligned}
\varphi(u, v, v, u, u+v, 0) & \leq 0 \\
& \Rightarrow u-q \frac{v+u}{2} \leq 0 \\
& \Rightarrow u \leq \frac{q}{2-q} v=h v
\end{aligned}
\end{aligned}
$$

where $h=\frac{q}{2-q}, 0<h<1$.

$\left(\varphi_{3}\right) \varphi(u, u, 0,0,0, u)=u-q u=(1-q) u \geq 0$ for all $u>0$.

\section{Main Results}

Theorem 1. Let $(X, d)$ be a metric space. Let $f, g: X \rightarrow X$ and $F, G$ : $X \rightarrow C B(X)$ be mappings satisfying the following conditions:

(1.1) $F(X) \subseteq f(X), G(X) \subseteq g(X)$,

$\varphi\left\{\begin{array}{c}H(F x, G y), d(f y, g x), D(f y, F x), \\ D(g x, G y), D(f y, G y), D(g x, F x)\end{array}\right\} \leq 0$ for all $x, y \in X$, where $\varphi \in \Phi$,

(1.3) the pairs $\{G, f\}$ and $\{F, g\}$ satisfy property $(E . A)$, then

(i) there exists $u, v \in X$ such that $f u \in G u$ and $g v \in F v$.

Further if

(1.4) $f$ is $G$-weakly commuting and $g$ is F-weakly commuting at their coincidence point, then

(ii) if $f u=g v=z \in X$, then $f z \in G z$ and $g z \in F z$,

(iii) if $f z=g z$ then $f z=g z \in F z \cap G z$,

(iv) if $f z=g z=z$, then $z$ is a common fixed point of $f, g, F$ and $G$.

Proof. Since the pair $\{F, g\}$ satisfies property (E.A), there is a sequence $\left\{x_{n}\right\}$ in $X$ such that

$$
\lim _{n \rightarrow \infty} g x_{n}=t \in A=\lim _{n \rightarrow \infty} F x_{n}
$$

for some $t \in X$ and $A \in C B(X)$.

Since $F(X)$ is closed, $t \in F(X)$. Therefore by (1.1) there exists $u \in X$ such that $t=f u$.

By (1.2), we have

$$
\varphi\left\{\begin{array}{c}
H\left(F x_{n}, G u\right), d\left(f u, g x_{n}\right), D\left(f u, F x_{n}\right), \\
D\left(g x_{n}, G u\right), D(f u, G u), D\left(g x_{n}, F x_{n}\right)
\end{array}\right\} \leq 0 .
$$


Letting $n \rightarrow \infty$, we get

$$
\begin{aligned}
& \varphi\{H(A, G u), 0, D(f u, A), D(f u, G u), D(f u, G u), D(f u, A)\} \\
= & \varphi\{H(A, G u), 0,0, D(f u, G u), D(f u, G u), 0\} \\
\leq & 0 .
\end{aligned}
$$

Since $f u \in A$, it follows from the definition of Hausdorff metric that

$$
\varphi\{D(f u, G u), 0,0, D(f u, G u), D(f u, G u), 0\} \leq 0 .
$$

Using $\left(\varphi_{a}\right)$, we get $D(f u, G u) \leq 0$, which is a contradiction. Hence $f u \in G u$.

Similarly, since the pair $\{G, f\}$ satisfies property $(E . A)$, there is a sequence $\left\{y_{n}\right\}$ in $X$ such that

$$
\lim _{n \rightarrow \infty} f y_{n}=q \in M=\lim _{n \rightarrow \infty} G y_{n}
$$

for some $q \in X$ and $M \in C B(X)$.

Since $G(X)$ is closed, $q \in G(X)$. Therefore by (1.1), there exists $v \in X$ such that $q=g v$.

By (1.2), we have

$$
\varphi\left\{\begin{array}{c}
H\left(F v, G y_{n}\right), d\left(f y_{n}, g v\right), D\left(f y_{n}, F v\right), \\
D\left(g v, G y_{n}\right), D\left(f y_{n}, G y_{n}\right), D(g v, F v)
\end{array}\right\} \leq 0 .
$$

Letting $n \rightarrow \infty$, we get

$$
\begin{aligned}
& \varphi\{H(F v, M), 0, D(g v, F v), D(g v, M), D(g v, M), D(g v, F v)\} \\
= & \varphi\{H(F v, M), 0, D(g v, F v), 0,0, D(g v, F v)\} \\
\leq & 0 .
\end{aligned}
$$

Since $g v \in M$, it follows from the definition of Hausdorff metric that

$$
\varphi\{D(g v, F v), 0, D(g v, F v), 0,0, D(g v, F v)\} \leq 0 .
$$
Fv.

Using $\left(\phi_{b}\right)$, we get $D(g v, F v) \leq 0$, which is a contradiction. Hence $g v \in$

This proves $(i)$.

Now since $f$ is $G$-weakly commuting at $u \in X$ and $g$ is $F$-weakly commuting at $v \in X$, we have

$$
f f u \in G f u \text { and } g g v \in F g v .
$$

If $f u=g v=z \in X$, then

$$
f z \in G z \text { and } g z \in F z .
$$

If $f z=g z$, then

$$
f z=g z \in F z \cap G z .
$$

If $f z=g z=z$, then $z$ is the common fixed point of $f, g, F$ and $G$.

This proves the Theorem.

Theorem 1 and the Examples 3-8 imply the following: 
Corollary 1. Let $(X, d)$ be a metric space. Let $f, g: X \rightarrow X$ and $F, G$ : $X \rightarrow C B(X)$ be mappings satisfying the conditions (1.1), (1.3), (1.4) and the following:

(2.1) $H(F x, G y) \leq \alpha \max \left\{\begin{array}{c}\frac{d^{2}(f y, g x)+D^{2}(f y, F x)}{d(f y, g x)+D(f y, F x)} \\ D(f y, G y)+D(g x, F x), D(g x, G y)\end{array}\right\}$

for all $x, y \in X$, where $\alpha \in(0,1 / 2)$.

Then $f, g, F$ and $G$ have a common fixed point.

Corollary 2. Let $(X, d)$ be a metric space. Let $f, g: X \rightarrow X$ and $F, G$ : $X \rightarrow C B(X)$ be mappings satisfying the conditions (1.1), (1.3), (1.4) and the following:

(3.1) $H(F x, G y)$

$$
\begin{aligned}
& \leq \alpha \frac{\max \{D(g x, G y)-d(f y, g x), D(g x, G y)-D(f y, F x)\}}{1+D(f y, G y) D(g x, F x)} \\
& +(1-\alpha) D(f y, G y) D(g x, F x) \\
& \text { for all } x, y \in X, \text { where } \alpha \in(0,1)
\end{aligned}
$$

Then $f, g, F$ and $G$ have a common fixed point.

Corollary 3. Let $(X, d)$ be a metric space. Let $f, g: X \rightarrow X$ and $F, G$ : $X \rightarrow C B(X)$ be mappings satisfying the conditions (1.1), (1.3), (1.4) and the following:

(4.1) $H^{p}(F x, G y)$

$\leq\left(\alpha d^{p}(f y, g x)+\beta\left(D^{p}(f y, F x)-D^{p}(g x, G y)\right)\right)$

$(1+D(f y, G y) D(g x, F x))$

for all $x, y \in X$, where $\alpha, \beta \in(0,1), \alpha+\beta<1$ and $p$ is an integer such that $p \geq 1$.

Then $f, g, F$ and $G$ have a common fixed point.

Corollary 4. Let $(X, d)$ be a metric space. Let $f, g: X \rightarrow X$ and $F, G$ : $X \rightarrow C B(X)$ be mappings satisfying the conditions (1.1), (1.3), (1.4) and the following:

(5.1) $2 H(F x, G y)$

$$
\begin{aligned}
& \leq \frac{\max \{d(f y, g x), D(g x, G y), D(f y, G y)\}}{1+(d(f y, g x)-D(f y, F x)) D(g x, G y)}-a D(f y, G y) \\
& +D(f y, F x) D(g x, F x) \\
& \text { for all } x, y \in X, \text { where } a \in(0,1) .
\end{aligned}
$$

Then $f, g, F$ and $G$ have a common fixed point.

Corollary 5. Let $(X, d)$ be a metric space. Let $f, g: X \rightarrow X$ and $F, G$ : $X \rightarrow C B(X)$ be mappings satisfying the conditions (1.1), (1.3), (1.4) and the following:

$$
\text { (6.1) } \begin{aligned}
& \beta H^{p}(F x, G y) \\
\leq & \beta\left(d^{p}(f y, g x)+D^{p}(g x, F x)\right)
\end{aligned}
$$




$$
-\frac{\alpha \max \left\{d^{p}(f y, g x)-D^{p}(f y, F x), D^{p}(g x, G y)\right\}}{1+\left(\alpha D^{p}(g x, G y)+\beta D^{p}(f y, G y)\right)\left(d^{p}(f y, g x)-D^{p}(f y, F x)\right)}
$$

for all $x, y \in X$, where $\beta \in(0,1), \alpha \geq 1$ and $p$ is an integer such that $p \geq 1$.

Then $f, g, F$ and $G$ have a common fixed point.

Corollary 6. Let $(X, d)$ be a metric space. Let $f, g: X \rightarrow X$ and $F, G$ : $X \rightarrow C B(X)$ be mappings satisfying the conditions (1.1), (1.3), (1.4) and the following:

$$
\text { (7.1) } H(F x, G y) \leq q \max \left\{\begin{array}{c}
d(f y, g x), \frac{D(f y, F x)+D(g x, G y)}{2}, \\
\frac{D(f y, G y), D(g x, F x)}{2}
\end{array}\right\}
$$

for all $x, y \in X$, where $q \in(0,1)$.

Then $f, g, H$ and $G$ have a common fixed point.

Example 9. Let $X=[0,4)$ with the usual metric $d$. Define the mappings $f, g: X \rightarrow X$ and $F, G: X \rightarrow C B(X)$ by

$$
\begin{array}{ll}
f x=\left\{\begin{array}{ll}
x, & 0 \leq x \leq 2 \\
\frac{x+3}{4}, & 2<x<4
\end{array},\right. & F x= \begin{cases}{\left[0, \frac{x}{3}\right],} & 0 \leq x \leq 2 \\
{\left[1, \frac{x+12}{8}\right],} & 2<x<4\end{cases} \\
g x= \begin{cases}\frac{x}{2}, & 0 \leq x \leq 2 \\
\frac{x+1}{2}, & 2<x<4\end{cases}
\end{array}
$$

and the $\operatorname{map} \varphi: \mathbb{R}_{+}^{6} \rightarrow \mathbb{R}$ such that

$$
\varphi\left(t_{1}, t_{2}, t_{3}, t_{4}, t_{5}, t_{6}\right)=t_{1}-\max \left\{t_{2}, \frac{t_{3}+t_{4}}{2}, \frac{t_{5}+t_{6}}{2}\right\}
$$

where

$$
\begin{array}{lll}
t_{1}=H(F x, G y), & t_{2}=d(f y, g x), & t_{3}=D(f y, F x), \\
t_{4}=D(g x, G y), & t_{5}=D(f y, G y), & t_{6}=D(g x, F x) .
\end{array}
$$

Then

1. $F(X) \subseteq f(X), G(X) \subseteq g(X)$.

2. The condition (2) is satisfied.

3. Consider the sequence $\left\{x_{n}\right\}$ in $X$ defined by $x_{n}=2+\frac{1}{n}, n=$ $1,2,3, \ldots$ Then

$$
\lim _{n \rightarrow \infty} f x_{n}=\frac{5}{4} \in \lim _{n \rightarrow \infty} G x_{n}=\left[1, \frac{5}{2}\right]
$$

and

$$
\lim _{n \rightarrow \infty} g x_{n}=\frac{3}{2} \in \lim _{n \rightarrow \infty} F x_{n}=\left[1, \frac{7}{4}\right] \text {. }
$$


Therefore the pairs $\{G, f\}$ and $\{F, g\}$ satisfy the property $(E . A)$.

4. $f$ is $G$-weakly commuting at the coincidence point 0 and $g$ is $F$-weakly commuting at the coincidence point 0 .

Therefore all the conditions of the Theorem 1 are satisfied and 0 is the common fixed point of $f, g, F$ and $G$.

If we put $g=f$ in the Theorem 1 , we get the following:

Corollary 7. Let $(X, d)$ be a metric space. Let $f: X \rightarrow X$ and $F, G: X \rightarrow$ $C B(X)$ be mappings satisfying the following conditions:

(8.1) $F(X) \subseteq f(X), G(X) \subseteq f(X)$,

(8.2) $\varphi\left\{\begin{array}{c}H(F x, G y), d(f y, f x), D(f y, F x), \\ D(f x, G y), D(f y, G y), D(f x, F x)\end{array}\right\} \leq 0$

for all $x, y \in X$, where $\varphi \in \Phi$,

(8.3) the pairs $\{G, f\}$ and $\{F, f\}$ satisfy property (E.A).

Then

(i) there exists $u, v \in X$ such that $f u \in G u$ and $f v \in F v$.

Further if,

(8.4) $f$ is $G$-weakly commuting and F-weakly commuting at their coincidence point, then

(ii) if $f u=f v=z \in X$, then $f z \in F z \cap G z$,

(iii) if $f z=z$, then $z$ is a common fixed point of $f, F$ and $G$.

If we put $F=G$ in Corollary 2, we get the following:

Corollary 8. Let $(X, d)$ be a metric space. Let $f: X \rightarrow X$ and $G: X \rightarrow$ $C B(X)$ be mappings satisfying the following conditions:

(9.1) $G(X) \subseteq f(X)$,

(9.2) $\varphi\left\{\begin{array}{c}H(G x, G y), d(f y, g x), D(f y, G x), \\ D(g x, G y), D(f y, G y), D(g x, G x)\end{array}\right\} \leq 0$

for all $x, y \in X$, where $\varphi \in \Phi$,

(9.3) the pair $\{G, f\}$ satisfies property (E.A).

Then

(i) there exists $u \in X$ such that $f u \in G u$.

Further if,

(9.4) $f$ is $G$-weakly commuting at their coincidence point, then

(ii) if $f u=z \in X$, then $f z \in G z$,

(iii) if $f z=z$, then $z$ is a common fixed point of $f$ and $G$.

Since a non-compatible hybrid pair satisfies the property $(E . A)$, we have the following:

Theorem 2. Let $(X, d)$ be a metric space. Let $f, g: X \rightarrow X$, and $F, G$ : $X \rightarrow C B(X), \varphi \in \Phi$ be mappings satisfying (1.1) and (1.2). If the pairs $\{G, f\}$ and $\{F, g\}$ are non-compatible, then $(i)$ of the Theorem 1 holds. Further if (1.4) is satisfied then (ii), (iii) and (iv) of the Theorem 1 hold. 
Conclusion. To prove common fixed point theorems for two hybrid pairs of mappings using the property (E.A), the condition $f u=g v=z \in X$ of Theorem 1 is essential. If we drop the property $(E . A)$ and assume $(X, d)$ as complete metric space, then the Theorem 1 can be proved without using $f u=g v=z \in X$.

\section{REFERENCES}

[1] A.J. Asad, Z. Ahmad, Common fixed of multivalued mappings with weak commutativity conditions, Radovi, Math. 9(1999), 119-124.

[2] B. Fisher, Mappings satisfying rational inequality, Nanta Math. 12(1979), 195-199.

[3] G. Jungck, B.E. Rhoades, Fixed point for set valued functions without continuity, Ind. J. Pure and Appl. Math. 29(3)(1998), 227-238.

[4] G. Jungck, Compatible mappings and common fixed points, Amer. Math. Monthly 83(1976), 261-263.

[5] G. Jungck, Compatible mappings and common fixed points, Int. J. Math. Math. Sci. 9(1986), 771-779.

[6] H. Kaneko, A common fixed point of weakly commuting multivalued mappings, Math. Japon 33(5)(1988), 741-744.

[7] H. Kaneko, S. Sessa, Fixed point theorems for compatible multivalued and singlevalued mappings, Int. J. Math. Math. Sci. 12(2)(1989), 257-262.

[8] I. Kubiaczyk, B. Deshpande, Coincidence point for noncompatible multivalued maps satisfying an implicit relation, Demonstratio Mathematica XXXIX (4)(2006).

[9] I. Kubiaczyk, N. Mostafa, A multivalued fixed point theorem in non-Archi-medean vector spaces, Novi Sad J. Math. 26(2)(1996), 11-115.

[10] M. Aamri, Moutawakil, Some new common fixed point theorems under strict contractive conditions, J. Math. Anal. Appl. 270(2002), 181-188.

[11] M. Abbas, D. Gopal, S. Radenović, A note on recently introduced commutative conditions, Indian Journal of Mathematics, 2012, in press.

[12] N. Shahzad, T. Kamran, Coincidence points and R-weakly commuting maps, Arch. Math. (Brno) 37(2001), 179-183.

[13] R.P. Pant, Common fixed point theorems for contractive maps, J. Math. Anal. Appl. 236(1998), 251-258.

[14] R.P. Pant, Common fixed points of Lipschitz type mappings pairs, J. Math. Anal. Appl. 240(1999), 280-283.

[15] R.P. Pant, Common fixed points of non-commuting mappings, J. Math. Anal. Appl. 188(1994), 436-440.

[16] R.P. Pant, Discontinuity and fixed points, J. Math. Anal. Appl. 240(1999), 284-289.

[17] S. B. Nadler, Multivalued contractions mappings, Pacific J. Math. 30(2)-(1969), 475488.

[18] S. Ithoh, W. Takahashi, Single valued mappings, multivalued mappings and fixed point theorems. J. Math. Anal. Appl. 59(1977), 514-521. 
[19] S. Kyzyska, I. Kubiaczyk, Fixed point theorems for upper semicontinuous and weakly upper semicontinuous multivalued mappings, Math. Japon 47(2)-(1998), 237-240.

[20] S. Sharma, B. Deshpande B., S. Chouhan , Fixed points for two hybrid pairs of mappings satisfying some weaker conditions on noncomplete metric spaces, SEA Bull. Math., accepted for publication.

[21] S. Sharma, B. Deshpande, Compatible multivalued mappings satisfying an implicit relation, SEA Bull. Math. 30(2006), 535-540.

[22] S.L.Singh, S.N. Mishra, Coincidence and fixed points of non self hybrid contraction, J. J. Math. Anal. Appl. 256(2001), 486-497.

[23] S.Sessa, M.S. Khan, Some remarks in best approximation theory, Math. J. Toyoma Univ. 17(1994), 151-165.

[24] S.Sessa, On weak commutativity condition of mappings in fixed point consideration, Publ. Inst. Math. 32(46)(1982), 149-155.

[25] T. Kamran, Coincidence and fixed points for hybrid strict contractions, J. Math. Anal. Appl. 299(2004), 235-241.

[26] T. Kubiak, Fixed point theorems for contractive type multivalued mappings, Math. Japon 30(1985), 89-101.

Bhavana Deshrande

Department of Mathematics

Govt. Arts and Science P.G. College

RATLAM-457001, M.P.

INDIA

E-mail address: bhavnadeshpande@yahoo.com

\section{Rohit Pathak}

Department of Applied Sciences

Institute of Engineering And Technology, DAVV

INDORE-452017, M.P.

INDIA

E-mail address: rohitpathakres@yahoo.in 